\title{
Narrative review of cardiac computed tomography perfusion: insights into static rest perfusion
}

\author{
Bruna Punzo $^{1}$, Carlo Cavaliere ${ }^{1}$, Erica Maffei ${ }^{2}$, Eduardo Bossone $^{3}$, Luca Saba $^{4}$, Filippo Cademartiri $^{1}$ \\ ${ }^{1}$ Department of Radiology, SDN IRCCS, Naples, Italy; ${ }^{2}$ Department of Radiology, Area Vasta 1, ASUR Marche, Urbino (PU), Italy; ${ }^{3}$ Department of \\ Cardiology, Ospedale Cardarelli, Naples, Italy; ${ }^{4}$ Department of Radiology, University of Cagliari, Cagliari, Italy \\ Contributions: (I) Conception and design: B Punzo, F Cademartiri; (II) Administrative support: F Cademartiri; (III) Provision of study materials or \\ patients: B Punzo, C Cavaliere; (IV) Collection and assembly of data: None; (V) Data analysis and interpretation: None; (VI) Manuscript writing: All \\ authors; (VII) Final approval of manuscript: All authors. \\ Correspondence to: Prof. Dr. Filippo Cademartiri, MD, PhD. Chairman, Department of Radiology, Area Vasta 1, ASUR Marche, Ospedale Civile "Santa \\ Maria della Misericordia” di Urbino, viale Federico Comandino, 70-61029, Urbino (PU), Italy. Email: filippocademartiri@gmail.com.
}

\begin{abstract}
Cardiac or left ventricular perfusion performed with cardiac computed tomography (CCT) is a developing method that may have the potential to complete in a very straight forward way the assessment of ischemic heart disease by means of CT. Myocardial CT perfusion (CTP) can be achieved with a single static scan during the first-pass of the iodinate contrast agent, with the monoenergetic or dual-energy acquisition, or as a dynamic, time-resolved scan during stress by using coronary vasodilator agents. Several methods can be performed, and we focused on static perfusion. CTP may serve as a useful adjunct to coronary CT angiography (CTA) to improve specificity of detecting myocardial ischemia. Technological advances will reduce the radiation dose of myocardial CTP, such as low tube voltage imaging or new reconstruction algorithms, making it a more viable clinical option. The advantages of static first-pass non-stress perfusion are several; the main one is that it can be done to each and every patient who undergoes CCT for the assessment of coronary artery tree. Future advances in CTP will likely improve the diagnostic accuracy of CTP + CTA, and will better estimate the severity of ischemia Therefore, it is simple and comprehensive. However, it has several limitations. In this review we will discuss the technique with its advantages and limitations.
\end{abstract}

Keywords: Cardiac computed tomography (CCT); perfusion; static; first-pass; stenosis; ischemia; accuracy

Submitted Jun 03, 2020. Accepted for publication Aug 10, 2020.

doi: $10.21037 / \mathrm{cdt}-20-552$

View this article at: http://dx.doi.org/10.21037/cdt-20-552

\section{Introduction}

Cardiac computed tomography (CCT) is the non-invasive reference model for the evaluation of coronary arteries (1) and has become the predominant imaging modality to identify coronary artery disease $(\mathrm{CAD})$ with high diagnostic accuracy. CT imaging of the heart has improved rapidly, and it is now possible to perform a comprehensive evaluation using a low amount of radiation dose.

Non-invasive acquisition of patients with suspected CAD has traditionally rested on two pillars: morphologic assessment for coronary artery stenosis and functional/ perfusion evaluation for determining the downstream hemodynamic significance of lesions (2). This approach is of fundamental importance as the morphological evaluation of coronary stenoses does not linearly reflect the extension and severity of myocardial ischemia throughout the left ventricle (LV).

Furthermore, by evaluating morphology and function in a combined way, the prognosis and supervision of patients are more reliable and efficient $(3,4)$.

According to the current appropriate use criteria and guidelines, CCT is the method of choice for the exclusion of significant coronary artery stenosis in patients with low 
and intermediate CAD risk profiles. Sensitivity (96\%) and specificity (86\%) of computed tomography angiography (CTA) determine its diagnostic accuracy $(5,6)$.

Moreover, the electrocardiogram (ECG) trigger acquisition allows us to quickly obtain functional parameters of the LV. Besides, this emerging technology is used for the evaluation of myocardial perfusion has the potential to become the standalone method for the evaluation of patients with suspected CAD, with a single modality and within a single imaging session.

Myocardial CT perfusion (CTP) imaging can implement information to CCT and is particularly useful in patients with heavily calcified coronary arteries or coronary artery stents (7).

Indeed, myocardial CTP can be achieved with a single static scan during the first-pass of the contrast agent, with the monoenergetic or dual-energy acquisition, or as a dynamic, time-resolved scan during stress by using coronary vasodilator agents such as adenosine or dipyridamole (8).

The goal of this article is to review the expanding body of literature on the CT estimation of myocardial perfusion focusing on static first-pass rest acquisition methods.

We present the following article in accordance with the Narrative Review reporting checklist (available at http:// dx.doi.org/10.21037/cdt-20-552).

\section{Myocardial perfusion methodologies}

Myocardial perfusion can be assessed through different imaging modalities. CCT can be considered a semiquantitative/quantitative method useful in detecting ischemia, and comparable to other perfusion imaging tests.

CCT may be considered in patients with low or intermediate pre-test probability of CAD and nonconclusive stress test. The advantage of CCT over other methods is to detect anatomical details of coronary tree and myocardium. The spatial resolution is very high compared to other imaging modalities but one of the disadvantages is the radiation dose exposure which, however, is gradually improving thanks to technological advancement.

Besides to radiological diagnostics, nuclear cardiology techniques play a main role in the evaluation of heart failure. Myocardial single photon emission computed tomography (SPECT) offer valuable information of ventricular function and myocardial perfusion and commonly used radio-tracers include technetium (Tc)-based agents, such as ${ }^{99 \mathrm{~m}} \mathrm{Tc}$-sestamibi and ${ }^{99 \mathrm{~m}} \mathrm{Tc}$-tetrofosmin. Moreover, positron emission tomography (PET) assess myocardial perfusion, metabolism and viability with high quality images and provide to quantitative analysis. Cardiac PET perfusion radio-tracers are rubidium- $82\left({ }^{82} \mathrm{Rb}\right)$, oxygen- 15 water $\left({ }^{15} \mathrm{O}-\mathrm{H}_{2} \mathrm{O}\right)$ and nitrogen-13 ammonia $\left({ }^{13} \mathrm{~N}-\mathrm{NH}_{3}\right)$, most often used with metabolic ${ }^{18} \mathrm{~F}$-fluorodeoxyglucose $\left({ }^{18} \mathrm{~F}-\mathrm{FDG}\right)(9)$.

Although nuclear medicine investigations represent, in the case of PET, the gold standard in the evaluation of myocardial metabolism, CTP has numerous advantages, well described in the next paragraph.

The limitations are in sharp decrease thanks to technological progress and depend largely on the equipment. Dose values delivered will therefore be different based on the performance and technology used.

CTP derives from the CTA acquisition but still remains affected to artifacts, such as beam hardening, which can be overcome thanks to the development of iterative reconstruction algorithms.

Despite the use of multiple protocols and various artifacts, the available data suggests than CTP may have an accuracy which is comparable to SPECT for identifying myocardial ischemia. Ultimately, CTP may serve as a useful adjunct to coronary CTA to improve specificity of detecting myocardial ischemia. Future advances in CTP will likely improve the diagnostic accuracy of CTP + CTA, and will better estimate the severity of ischemia (10).

\section{Overview of myocardial CTP}

CT evaluation of myocardial perfusion is based on the distribution of iodinated contrast agent during its first pass over the myocardium. Because the arterial blood flow determines the contrast material's distribution, myocardial perfusion defects can be identified as hypoattenuating areas containing less concentration of contrast material (11).

Static CTP imaging occurs at or near the peak contrast opacification of the left ventricle and includes the acquisition of a single dataset. Dynamic CTP imaging requires sequential datasets during the initial pass of iodinated contrast from the venous to the arterial circulation. On both static and dynamic CTP imaging, regions of LV hypoperfusion will appear as low attenuation regions within an otherwise normal contrast distribution (i.e., perfusion), typically worse in the subendocardial layer than the epicardial layer.

A comprehensive myocardial CTP examination should include both rest and stress acquisitions to differentiate reversible from fixed myocardial perfusion defects (12). 

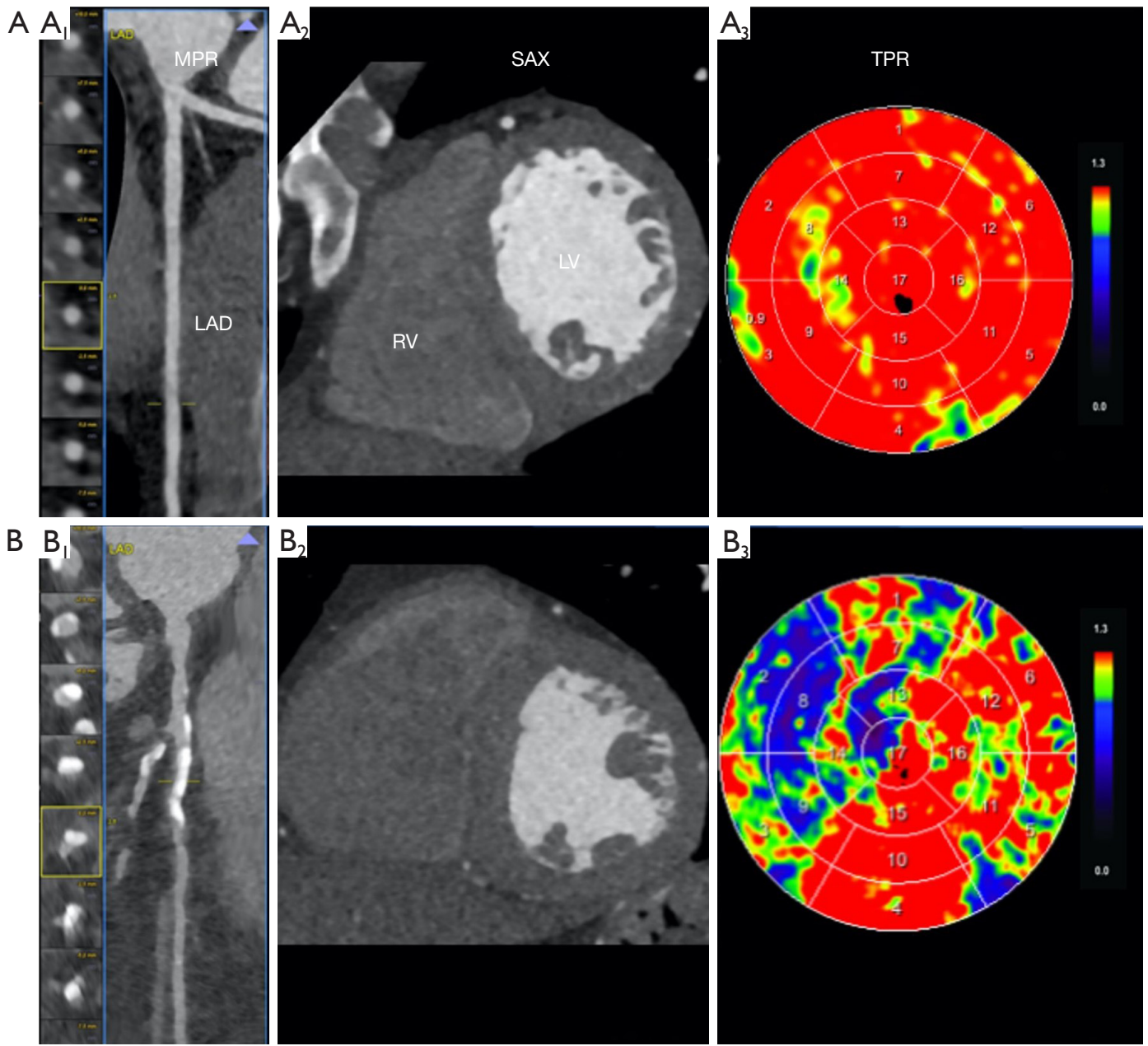

Figure 1 Examples of static perfusion with CCT. Panel A $\left(A_{1}-A_{3}\right)$, shows a normal perfusion pattern in a patient with normal left anterior descending coronary artery (LAD), on multiplanar reformat images $\left(\mathrm{A}_{1} ; \mathrm{MPR}\right)$, on short axis $\left(\mathrm{A}_{2} ; \mathrm{SAX}\right)$ of the left ventricle $(\mathrm{LV})$, and on the perfusion mask of transmural perfusion ratio (TPR) $\left(\mathrm{A}_{3}\right)$. On SAX and TPR every left ventricular segment shows normal thickness and morphology with homogeneous first-pass perfusion. Panel $B\left(B_{1}-B_{3}\right)$, shows a patient with a severe calcified LAD stenosis $\left(B_{1}\right.$; MPR); even though thickness and morphology of anterolateral segments of the $\mathrm{LV}$ appear normal $\left(\mathrm{B}_{2} ; \mathrm{SAX}\right)$, there is a quite large first-pass perfusion defect (or delay) on TPR $\left(\mathrm{B}_{3}\right)$. CCT, cardiac computed tomography.

Rest acquisition is derived from datasets obtained for coronary anatomy (13). It is less sensitive to the detection of myocardial ischemia since blood flow, and therefore perfusion is compromised when the stenosis is hemodynamically significant and exceeds $85-90 \%$ of the luminal diameter. A subendocardial or transmural hyperenhancement in the rest phase may indicate a critical flow-limiting coronary artery stenosis or a myocardial necrosis/scar from infarction.

Moreover, being derived from the CCT acquisition, it does not require further scans, thus decreasing the radiation dose delivered to the patient.
Besides, available post-processing software may allow for the generation of attenuation-based color mapping and a semi-quantitative assessment using a transmural perfusion ratio (TPR). TPR is just the ratio of the mean Hounsfield unit (HU) of a region of interest (ROI) within the subendocardial layer compared with the mean HU attenuation within the same ROI of the epicardial layer (Figure 1). This evaluation highlights the well-described aspects of an ischemic wave, which is worse in the subendocardial myocardial layers and gradually improves moving closer to the epicardial coronaries (i.e., creating a gradient). The use of TPR in static CTP significantly improves 
diagnostic accuracy compared to other techniques (14).

Other CT imaging signs of necrosis/fibrosis may be identified at the rest phase, such as myocardial wall thinning, lipomatous metaplasia $(<10 \mathrm{HU})$, myocardial calcifications, aneurysmal/pseudoaneurysmal dilation, and mural thrombus.

These data highlight the significance of rest imaging in myocardial perfusion evaluation (12).

\section{Acquisition techniques and image analysis}

Static CTP imaging allows for qualitative evaluation of myocardial iodine contrast attenuation of a single acquisition. This image is taken during the arterial phase of first-pass contrast enhancement. The flow rate of injected contrast medium should be at least $5 \mathrm{~mL} / \mathrm{s}$, to provide for notable differences in contrast enhancement. It is of paramount importance the timing for first-pass single-phase stress CTP to increase diagnostic accuracy (15). Because the same acquisition can be used for the evaluation of coronary artery stenosis and myocardial blood supply, static CTP at rest can be achieved employing any CT scanner able to obtain diagnostic-quality CCT (16), hence being less technology-dependent.

Static CTP evaluation at rest is generally performed using CCT image datasets. Thus, no additional acquisition time or radiation is necessary. It can further be categorized into single- and dual-energy techniques.

\section{Single energy acquisition}

Generally, the CCT examination allows generating rest myocardial CTP imaging ECG-gating of coronary CTA or CTP can be retrospective but also prospective, which is another feature of most advanced multislice CT scanners (64 or more slices), providing a significant reduction in terms of radiation dose (less than $5 \mathrm{mSv}$ ), without any significant decreasing in image quality (17).

A prospective ECG-triggered high-pitch spiral acquisition performed with the 2 nd-generation 128 -slice dual-source CT (DSCT) scanner concedes the volumetric acquisition data of the heart in a single cardiac cycle with radiation dose as low as $1 \mathrm{mSv}(18,19)$.

The analysis of myocardial perfusion is qualitative and consists in the identification of areas of hypodensity corresponding to areas of hypoperfusion. For myocardial perfusion analysis, multiplanar reconstructions should be carried out at 5 to $8 \mathrm{~mm}$ to improve the contrast-to- noise ratio (17). Myocardial contrast enhancement rises proportionally with iodine concentration, so perfusion defects appear as a hypodense area with subendocardial or transmural distribution concerning the normal myocardium (Figure 1).

Automated software providing modeling of the perfusion data into a $3 \mathrm{D}$ volume-rendering of the $\mathrm{LV}$ and a 17-segment polar map, are readily available.

Finally, automated software applications allow for evaluation of the TPR (an endocardial-to-mean epicardial ratio of attenuation values) using the 17 -segment bulls s-eye scheme. However, this semi-quantitative index parameter seems not reliable in the presence of prior infarction or significant beam-hardening. To better distinguish between artifacts and true perfusion defects, myocardial perfusion in multiphase reconstructions are recommended. Artifacts may affect only a single cardiac phase, whereas true defects should persist throughout the entire cardiac cycle (16). Data can be reconstructed with an iterative beam hardening correction (IBHC) algorithm to improve reliability. IBHC is a raw data-based beam hardening correction algorithm that raises image quality using 3D forward projection and exploits an additional two-compartment iodine/water model, to reduce beam hardening artifacts while having proper representation of anatomical structures (20).

The decisive move for the study of a CTP is the match of perfusion defects with the anatomic localization of coronary stenosis, which is crucial for understanding the hemodynamic significance of CAD $(8,13)$.

\section{Dual-energy acquisition}

Dual-energy computed tomography (DECT) was first introduced in the late 1970s and provided for the differentiation of materials based on their X-ray attenuation at different tube voltages. The DECT technique permits the simultaneous acquisition of CT data using two different photon spectra (at $80 / 90 / 100$ and $140 / 150 \mathrm{kV}$ ) of distinctly different mean energies within a single helical acquisition (21).

Based on the specific attenuation characteristics of the different tissues, DECT enables identifying the features of the tissue and evaluating the myocardial blood supply by mapping iodine distribution within the myocardium (22).

Different vendors propose specific CT technologies to perform dual-energy acquisitions. Dual X-ray source system (Siemens Healthineers) is the most commonly used technology: there are two independent tubes paired with two detectors that concurrently emit high (140-150 kV) and 
Table 1 Summary of main static perfusion studies with CCT

\begin{tabular}{|c|c|c|c|c|c|c|c|c|c|}
\hline Rest-static CTP studies & $\begin{array}{c}\text { No. of } \\
\text { subjects }\end{array}$ & CT technology & $\begin{array}{c}\text { CT perfusion } \\
\text { protocol }\end{array}$ & $\begin{array}{l}\text { CT dose } \\
\text { (mSv) }\end{array}$ & $\begin{array}{l}\text { Reference } \\
\text { technique }\end{array}$ & $\begin{array}{c}\text { Sensitivity } \\
(\%)\end{array}$ & $\begin{array}{c}\text { Specificity } \\
(\%)\end{array}$ & $\begin{array}{l}\text { PPV } \\
(\%)\end{array}$ & $\begin{array}{l}\text { NPV } \\
(\%)\end{array}$ \\
\hline Koonce et al. (23) & 36 & $1^{\text {st }}$-gen. DSCT & Rest & 14 & SPECT & 92 & 93 & 83 & 97 \\
\hline Wang et al. (29) & 31 & $1^{\text {st }}$-gen. DSCT & Rest & 10.5 & SPECT, ICA & 89 & 98 & 87 & 98 \\
\hline Kachenoura et al. (31) & 64 & 64-MDCT & Rest & $7-15$ & ICA & 96 & 68 & 88 & 87 \\
\hline George et al. (32) & 24 & 64-MDCT & Rest & 16.8 & SPECT, ICA & 86 & 92 & 92 & 85 \\
\hline
\end{tabular}

$1^{\text {st }}$ and $2^{\text {nd }}$ gen., generation of equipment; DSCT, dual source computed tomography; MDCT, multi-detector computed tomography; ICA, invasive coronary angiography; SPECT, single photon emission computed tomography; CCT, cardiac computed tomography; CTP, computed tomography perfusion; CT, computed tomography; PPV, positive predictive value; NPV, negative predictive value.

low (80-90-100 kV) energy levels (17).

A second modality is based on single-source CT with rapid (about $0.25 \mathrm{~ms}$ ) switching of tube voltage between 80 and $140 \mathrm{kV}$ either in a single gantry rotation (GSI Cardiac, GE Healthcare) or in sequential rotations (Acquilion One, Toshiba) $(13,17)$.

Another method is represented by the dual-layer ("sandwich") detector (Philips Healthcare). Two different materials can discriminate between low and high energy photons, with the source working at constant tube voltage; however, this system is not yet available in clinical practice (21).

DSCT-based dual-energy CT can generate an iodine distribution map. Because myocardial iodine concentration is a surrogate for myocardial perfusion, its volume reflects the myocardial blood pool (23). A color-coded map represents different myocardial territories and reflects iodine distribution in all segments, according to American Heart Association (AHA) classification (17 segments). Advanced post-processing workstations automatically compute perfusion maps, and then the relative normalized myocardial attenuation density and TPR are obtained.

DECT myocardial perfusion studies performed during rest or stress protocols showed good accuracy compared with various reference modalities (24-29).

Ruzsics et al. (25) studied the feasibility of rest myocardial perfusion imaging using a first-generation DECT system matched with SPECT in 36 patients and reported a sensitivity and specificity of $92 \%$ and $93 \%$, respectively.

A study of 31 patients showed improved sensitivity and specificity in detecting CAD by supplying coronary CTA with DECT myocardial perfusion (29).

De Cecco and colleagues (30) reported a significant increase in specificity from $33 \%$ to $67 \%$ when DECT myocardial perfusion was combined with coronary CTA when compared with invasive coronary angiography (31-33) (Table 1).

Like the single-energy static technique, DECT generates a snapshot of the myocardial iodine distribution at a single time point. As with single-energy perfusion imaging, different myocardial regions may be scanned in different phases of the iodine contrast uptake, thus narrowing the comparability of myocardial iodine content among regions. An additional limitation is that the non-standardized assessment of the iodine distribution maps makes the evaluation highly user-dependent. Beam hardening artifacts can compromise the correct evaluation of iodine distribution maps that could affect areas of myocardial hypoperfusion and so false-positive findings. The development of new reconstruction algorithms aims to prevent these artifacts that affecting the evaluation of myocardial perfusion, such as the IBHC mentioned above.

\section{Clinical implementation and role}

At present, a variety of protocols are available for myocardial perfusion imaging. Progress will reduce the radiation dose of myocardial CTP, such as low tube voltage imaging or new reconstruction algorithms, making it a more viable clinical option (7).

The rest static CTP technique has the potential to become the most widely used in the clinical setting, given the simplicity of execution, speed, and tolerability with a minimum dose delivery. In clinical routine, identifying normal coronary artery morphology and related normal myocardial perfusion may not require stress imaging. It 
is also possible for those patients diagnosed with critical stenosis of one of the main coronary vessels with resting perfusion defects, where invasive coronary angiography is indicated rather than stress imaging. Several studies have suggested that resting imaging is adequate in the evaluation of myocardial perfusion (32,34). Myocardial infarction can be recognized through low myocardial attenuation areas on resting CT images; however, rest-only protocols have lower diagnostic accuracy than rest-stress protocols (35).

A variety of other methods have also been improved to evaluate the functional signal of coronary artery stenosis by CT imaging. These include the CT-derived fractional flow reserve (36) and the transluminal attenuation gradient of the coronary artery (37).

These methods have the benefit that they are derived from rest images without the necessity of additional imaging, contrast medium, or radiation exposure. Further work is needed to determine whether these methods are better for myocardial perfusion imaging (7).

The main advantage of CT imaging is that accurate information on the coronary arteries can also be obtained. It is also helpful for patients where magnetic resonance imaging (MRI) is contraindicated. CTP imaging can be performed as an add on test after CTA if there is diagnostic doubt or it can be planned for patients with known or suspected heavy calcification or coronary artery stents. Also, CTP imaging can be performed in order to aid the planning of interventions.

\section{Radiation dose}

When considering radiation exposure, static CTP imaging is chosen over dynamic techniques.

The radiation dose for complete static CTP studies varies greatly depending on the specific acquisition protocol. Useful dose values between 2.5 and $21 \mathrm{mSv}$ have been reported with frequent radiation exposure of $12 \mathrm{mSv}$. Combined dynamic rest and stress CTP studies can require radiation exposure of approximately $18 \mathrm{mSv}$ (2). Also, from standard CCT acquisition, it is possible to obtain rest static CT myocardial perfusion images; this is not the case with dynamic imaging. In this case, no further dose is delivered to the patient.

Moreover, many factors may affect the radiation dose, such as the patient's biometrical parameters [body mass index (BMI), cardiac output, and heart rate], the type of CT equipment accessible, and the CT protocols used, which have to be tailored to the patient (17).
Current applications are directed to reduce radiation exposure while maintaining high diagnostic performance. In this interest, the use of recent technical findings, including the low voltages (70 to $80 \mathrm{kV}$ ) acquisition, automated tube current modulation, and iterative reconstruction, seems to be able to achieve this ambitious goal (13).

\section{Conclusions}

Current experiences suggest that adding perfusion imaging to the standard morphological imaging performed with CTA improves diagnostic accuracy and positive predictive value. It not only provides data on coronary artery atherosclerotic plaques but also functional and hemodynamic information on the myocardium. In various CTP protocols, the evaluation of perfusion is qualitative, quantitative, or semi-quantitative. Implementing this aspect could represent the turning point in the clinical routine to better stratify the patient based on risks and results obtained.

\section{Acknowledgments}

Funding: None.

\section{Footnote}

Provenance and Peer Review: This article was commissioned by the Guest Editor (Filippo Cademartiri) for the series "Clinical Impact of Cardiac CT in Clinical Practice" published in Cardiovascular Diagnosis and Therapy. The article was sent for external peer review organized by the Guest Editor and the editorial office.

Reporting Checklist: The authors have completed the Narrative Review reporting checklist. Available at http:// dx.doi.org/10.21037/cdt-20-552

Conflicts of Interest: All authors have completed the ICMJE uniform disclosure form (available at http://dx.doi. org/10.21037/cdt-20-552). The series "Clinical Impact of Cardiac CT in Clinical Practice" was commissioned by the editorial office without any funding or sponsorship. FC served as the unpaid Guest Editor of the series and serves as an unpaid editorial board member of Cardiovascular Diagnosis and Therapy from Jul 2019 to Jun 2021. LS serves as an unpaid editorial board member of Cardiovascular Diagnosis and Therapy from Jul 2019 to Jun 2021. The other 
authors have no other conflicts of interest to declare.

Ethical Statement: The authors are accountable for all aspects of the work in ensuring that questions related to the accuracy or integrity of any part of the work are appropriately investigated and resolved.

Open Access Statement: This is an Open Access article distributed in accordance with the Creative Commons Attribution-NonCommercial-NoDerivs 4.0 International License (CC BY-NC-ND 4.0), which permits the noncommercial replication and distribution of the article with the strict proviso that no changes or edits are made and the original work is properly cited (including links to both the formal publication through the relevant DOI and the license). See: https://creativecommons.org/licenses/by-nc-nd/4.0/.

\section{References}

1. Miller JM, Rochitte CE, Dewey M, et al. Diagnostic performance of coronary angiography by 64-row CT. N Engl J Med 2008;359:2324-36.

2. Varga-Szemes A, Meinel FG, De Cecco CN, et al. CT myocardial perfusion imaging. AJR Am J Roentgenol 2015;204:487-97.

3. Sarno G, Decraemer I, Vanhoenacker PK, et al. On the inappropriateness of noninvasive multidetector computed tomography coronary angiography to trigger coronary revascularization: a comparison with invasive angiography. JACC Cardiovasc Interv 2009;2:550-7.

4. Shaw LJ, Berman DS, Maron DJ, et al. Optimal medical therapy with or without percutaneous coronary intervention to reduce ischemic bur- den: results from the Clinical Outcomes Utilizing Revascularization and Aggressive Drug Evaluation (COURAGE) trial nuclear substudy. Circulation 2008;117:1283-91.

5. Taylor AJ, Cerqueira M, Hodgson JM, et al. ACCF/SCCT/ ACR/AHA/ASE/ASNC/NASCI/ SCAI/SCMR 2010 appropriate use criteria for cardiac computed tomography: a report of the American College of Cardiology Foundation Appropriate Use Criteria Task Force, the Society of Cardiovascular Computed Tomography, the American College of Radiology, the American Heart Association, the American Society of Echocardiography, the American Society of Nuclear Cardiology, the North American Society for Cardiovascular Imaging, the Society for Cardiovascular Angiography and Interventions, and the Society for Cardiovascular Magnetic Resonance.
Circulation 2010;122:e525-55.

6. Mordi I, Stanton T, Carrick D, et al. Comprehensive dobutamine stress CMR versus echocardiography in LBBB and suspected coronary artery disease. JACC Cardiovasc Imaging 2014;7:490-8.

7. Williams MC, Newby DE. CT myocardial perfusion imaging: current status and future directions. Clin Radiol 2016;71:739-49.

8. Cademartiri F, Seitun S, Clemente A, et al. Myocardial blood flow quantification for evaluation of coronary artery disease by computed tomography. Cardiovasc Diagn Ther 2017;7:129-50.

9. Angelidis G, Giamouzis G, Karagiannis G, et al. SPECT and PET in ischemic heart failure. Heart Fail Rev 2017;22:243-61.

10. Branch KR, Haley RD, Bittencourt MS, et al. Myocardial computed tomography perfusion. Cardiovasc Diagn Ther 2017;7:452-62.

11. George RT, Silva C, Cordeiro MA, et al. Multidetector computed tomography myocardial perfusion imaging during adenosine stress. J Am Coll Cardiol 2006;48:153-60.

12. Mehra VC, Valdiviezo C, Arbab-Zadeh A, et al. A stepwise approach to the visual interpretation of CTbased myocardial perfusion. J Cardiovasc Comput Tomogr 2011;5:357-69.

13. Seitun S, Castiglione Morelli M, Budaj I, et al. Stress Computed Tomography Myocardial Perfusion Imaging: A New Topic in Cardiology. Rev Esp Cardiol (Engl Ed) 2016;69:188-200.

14. Ramsey BC, Fentanes E, Choi AD, et al. Myocardial Assessment with Cardiac CT: Ischemic Heart Disease and Beyond. Curr Cardiovasc Imaging Rep 2018;11:16.

15. Bischoff B, Bamberg F, Marcus R, et al. Optimal timing for first-pass stress CT myocardial perfusion imaging. Int J Cardiovasc Imaging 2013;29:435-42.

16. Danad I, Szymonifka J, Schulman-Marcus J, et al. Static and dynamic assessment of myocardial perfusion by computed tomography. Eur Heart J Cardiovasc Imaging 2016;17:836-44.

17. Seitun S, De Lorenzi C, Cademartiri F, et al. CT Myocardial Perfusion Imaging: A New Frontier in Cardiac Imaging. Biomed Res Int 2018;2018:7295460.

18. Maffei E, Martini C, Rossi A, et al. Diagnostic accuracy of second-generation dual-source computed tomography coronary angiography with iterative reconstructions: a real-world experience. Radiol Med 2012;117:725-38.

19. Feuchtner G, Goetti R, Plass A, et al. Adenosine stress high-pitch 128-slice dual-source myocardial computed 
tomography perfusion for imaging of reversible myocardial ischemia: comparison with magnetic resonance imaging. Circ Cardiovasc Imaging 2011;4:540-9.

20. Forte E, Punzo B, Gentile F, et al. Normal patterns of left ventricle rest myocardial perfusion assessed by thirdgeneration cardiac computed tomography. Clin Physiol Funct Imaging 2020;40:30-6.

21. Danad I, Ó Hartaigh B, Min JK. Dual-energy computed tomography for detection of coronary artery disease. Expert Rev Cardiovasc Ther 2015;13:1345-56.

22. Yi Y, Jin ZY, Wang YN. Advances in myocardial CT per- fusion imaging technology. Am J Transl Res 2016;8:4523-31.

23. Koonce JD, Vliegenthart R, Schoepf UJ, et al. Accuracy of dual-energy computed tomography for the measurement of iodine concentration using cardiac CT protocols: validation in a phan- tom model. Eur Radiol 2014;24:512-8.

24. Ko SM, Choi JW, Song MG, et al. Myocardial perfusion imaging using adenosine-induced stress dual-energy computed tomography of the heart: comparison with cardiac magnetic resonance imaging and conventional coronary angiography. Eur Radiol 2011;21:26-35.

25. Ruzsics B, Schwarz F, Schoepf UJ, et al. Comparison of dual-energy computed tomography of the heart with single photon emission computed tomography for assessment of coronary artery stenosis and of the myocardial blood supply. Am J Cardiol 2009;104:318-26.

26. Kim SM, Chang SA, Shin W, et al. Dual- energy CT perfusion during pharmacologic stress for the assessment of myocardial per- fusion defects using a second-generation dual- source CT: a comparison with cardiac magnetic resonance imaging. J Comput Assist Tomogr 2014;38:44-52.

27. Ko SM, Choi JW, Hwang HK, et al. Diagnostic performance of combined noninvasive anatomic and functional assessment with dual-source CT and adenosineinduced stress dual-energy CT for detection of significant coronary stenosis. AJR Am J Roentgenol 2012;198:512-20.

28. Ko SM, Park JH, Hwang HK, et al. Direct comparison of stress- and rest-dual-energy computed tomography for detection of myocardial perfusion defect. Int J Cardiovasc Imaging 2014;30:41-53.

29. Wang R, Yu W, Wang Y, et al. Incremental value of dual-energy CT to coronary CT angiography for the detection of significant coronary stenosis: comparison with quantitative coronary angiography and single photon emission computed tomography. Int J Cardiovasc Imaging 2011;27:647-56.
30. De Cecco CN, Harris BS, Schoepf UJ, et al. Incremental value of pharmacological stress cardiac dual-energy CT over coronary CT angiography alone for the assessment of coronary artery disease in a high-risk population. AJR Am J Roentgenol 2014;203:W70-7.

31. Kachenoura N, Gaspar T, Lodato JA, et al. Combined assessment of coronary anatomy and myocardial perfusion using multidetector computed tomography for the evaluation of coronary artery disease. Am J Cardiol 2009;103:1487-94.

32. George RT, Arbab-Zadeh A, Miller JM, et al. Adenosine stress 64- and 256-row detector computed tomography angiography and perfusion imaging: a pilot study evaluating the transmural extent of perfusion abnormalities to predict atherosclerosis causing myocardial ischemia. Circ Cardiovasc Imaging 2009;2:174-82.

33. Osawa K, Miyoshi T, Koyama Y, et al. Additional diagnostic value of first- pass myocardial perfusion imaging without stress when combined with 64-row detector coronary CT angiography in patients with coronary artery disease. Heart 2014;100:1008-15.

34. Troupis JM, Karge A, Seneviratne S, et al. Myocardial density analysis utilizing automated myocardial defect analysis software on resting 320-detector MDCT. Int J Cardiovasc Imaging 2013;29:1121-7.

35. Meinel FG, De Cecco CN, Schoepf UJ, et al. Firstarterial-pass dual-energy CT for assessment of myocardial blood supply: do we need rest, stress, and delayed acquisition? Comparison with SPECT. Radiology 2014;270:708-16.

36. Hlatky MA, De Bruyne B, Pontone G, et al. Quality of life and economic outcomes of assessing fractional flow reserve with computed tomography angiography: the PLATFORM Study. J Am Coll Cardiol 2015;66:2315-23.

37. Wong DT, Ko BS, Cameron JD, et al. Comparison of diagnostic accuracy of combined assessment using adenosine stress CT perfusion (CTP) + computed tomography angiography (CTA) with transluminal attenuation gradient (TAG320) + CTA against invasive fractional flow reserve (FFR). J Am Coll Cardiol 2014;63:1904-12.

Cite this article as: Punzo B, Cavaliere C, Maffei E, Bossone E, Saba L, Cademartiri F. Narrative review of cardiac computed tomography perfusion: insights into static rest perfusion. Cardiovasc Diagn Ther 2020;10(6):1946-1953. doi: 10.21037/cdt$20-552$ 